\title{
The Performance of Universal Adhesives on Orthodontic Bracket Bonding
}

\author{
Muhittin Ugurlu Muhammed Hilmi Buyukcavus² \\ ${ }^{1}$ Department of Restorative Dentistry, Faculty of Dentistry, \\ Süleyman Demirel University, Isparta, Turkey \\ ${ }^{2}$ Department of Orthodontics, Faculty of Dentistry, Süleyman \\ Demirel University, Isparta, Turkey

\begin{abstract}
Address for correspondence Muhittin Ugurlu, DDS, Faculty of Dentistry, Süleyman Demirel University, East Campus, 32200, Isparta, Turkey (e-mail: dtmuhittinugurlu@gmail.com).
\end{abstract}

Eur J Gen Dent 2021;10:19-24.

\begin{abstract}
Keywords

- bracket

- bonding

- dental adhesives

- double application

- universal adhesive

Objective This article aimed to assess the effects of double application of universal adhesives on the shear bond strength of orthodontic brackets.

Materials and Method Seventy-five extracted human premolars were used. The teeth were randomly assigned into five groups based on the adhesive procedure $(n=15)$. The universal adhesives Scotchbond Universal (3M Oral Care) and Prime\&Bond Universal (Dentsply) were used (following manufacturer's instructions and double application). Transbond XT Primer (3M Unitek) was employed as control. Following adhesive application, the brackets were bonded on the tooth surfaces. After storage in distilled water for 24 hours at $37^{\circ} \mathrm{C}$, the specimens were subjugated to the shear bond strength test under a universal testing machine (Autograph AGS-X; Shimadzu). Data were analyzed with one-way analysis of variance and least significant difference tests $(p=0.05)$. The adhesive remnant index (ARI) was determined using a stereomicroscope (S4E; Leica Microsystems). Data of ARI scores were submitted to Pearson's chi-square test.

Results The highest shear bond strengths were acquired with Scotchbond Universal $(p<0.05)$. The double application of Scotchbond Universal did not impact the shear bond strength. The lowest shear bond strength was found in Prime\&Bond Universal $(p<0.05)$. The double application of Prime\&Bond Universal increased the shear bond strength $(p<0.05)$. There were no significant differences in ARI scores among the groups $(p>0.05)$.

Conclusion The universal adhesives may be an alternative for the bonding of orthodontic brackets. The double application of universal adhesives might improve the shear bond strength of orthodontic brackets depending on the material.
\end{abstract}

\section{Introduction}

The adequate bonding between teeth and brackets is one of the factors influencing the success of the fixed orthodontic treatment, which forms an important part of orthodontic practice. ${ }^{1-3}$ Adequate bond strength is very important to make a successful orthodontic treatment. ${ }^{4,5}$ The bond strength of the bracket depends on different factors, such as the bracket base, the adhesive material, and the preparation of tooth surface. ${ }^{1}$ The Transbond XT (3M Unitek, St. Paul, Minnesota, United States) etch-and-rinse adhesive system is one of the standard adhesive systems frequently used in orthodontic
DOI https://doi.org/ $10.1055 / \mathrm{s}-0041-1732778$ ISSN 2320-4753 (c) 2021. European Journal of General Dentistry.

This is an open access article published by Thieme under the terms of the Creative Commons Attribution-NonDerivative-NonCommercial-License, permitting copying and reproduction so long as the original work is given appropriate credit. Contents may not be used for commercial purposes, or adapted, remixed, transformed or built upon. (https://creativecommons.org/licenses/by-nc-nd/4.0/). Thieme Medical and Scientific Publishers Pvt. Ltd. A-12, 2nd Floor, Sector 2, Noida-201301 UP, India 
treatments. $^{2}$ This system provides adequate bond strength to resist masticatory and other forces in the oral environment. ${ }^{2,6}$

The developments in adhesive dentistry aim to ease bonding procedures through decreasing application steps, abridging clinical application time, and reducing technique sensitivity. ${ }^{7}$ Clinicians want to use only one adhesive for all cases and shorten the application time. The latest generation of adhesives is so-called universal or multimode adhesives that may use in any bonding strategy, including etch-and-rinse, self-etch, and selective enamel etching. ${ }^{8}$ The manufacturers state that universal adhesives can be employed for the placement of both direct and indirect restorations, including metals, zirconia, porcelain, and composite. A previous study concluded that the low shear bond strength was obtained for orthodontic brackets, which were bonded by universal adhesives in self-etch mode. ${ }^{3}$ However, it has been stated that the shear bond strength of orthodontic brackets bonded with a universal adhesive could be increased by supplement, an initial acid etching or laser conditioning step. ${ }^{6}$ Moreover, it has been shown that the universal adhesives could provide sufficient bond strength for orthodontic bracket bonding to composite, ceramic, zirconia, and porcelain surfaces..$^{9-12}$ There is not enough result regarding the efficacy of universal adhesives in the bonding of orthodontic brackets.

The universal adhesives had a rather thin film thickness because high solvent content requires more evaporation by air blowing/thinning, thus causing low bond strength. ${ }^{13,14}$ It has been revealed that the double application of universal adhesives was effective in improving the microtensile bond strength of dentin ${ }^{13,15}$ and shear bond strength of enamel. ${ }^{16}$ The enhanced bond strength by the double application has been attributed to an increase in adhesive layer thickness. ${ }^{17-19}$ The thicker adhesive layer might absorb stresses cumbered on the adhesive interface, including polymerization shrinkage stresses and enhance stress distribution during testing. ${ }^{20,21}$ Nevertheless, the effect of the double application of universal adhesives on the shear bond strength of orthodontic brackets has not also been tested so far.

Wherefore, the purpose of the present study was to evaluate the effects of the double application of universal adhesives on the shear bond strength of orthodontic brackets. The null hypotheses to be tested were (1) that there would not be significant differences in shear bond strength between the universal adhesives and Transbond XT, and (2) that the double application of universal adhesives would not improve the shear bond strength of orthodontic brackets.

\section{Materials and Methods}

Seventy-five human premolars which were extracted for orthodontic purposes were employed following ethical approval (ref no: 2019/327). The teeth had no endodontic treatment, carious lesions, restorations, enamel defects, and visible cracks. The teeth were kept in $0.5 \%$ chloramine-T solution at $4^{\circ} \mathrm{C}$ and employed within 3 months following extraction. The teeth were checked for the absence of cracks and defects under a stereomicroscope (S4E; Leica Microsystems, Wetzlar, Germany). After the cleaning of the buccal surfaces of teeth using a rubber cap and slurry of nonfluoridated pumice, they were fixed on acrylic resin blocks. The buccal enamel surface of each tooth was treated with $37 \%$ orthophosphoric acid (Transbond XT Etching Gel; 3M Unitek) for 15 seconds, water rinsed, and air-dried. The teeth were randomly assigned into five groups based on the adhesive procedures ( $n=15$ ).

The two universal adhesive systems were tested: Scotchbond Universal Adhesive (3M Oral Care, St. Paul, Minnesota, United States) and Prime\&Bond Universal (Dentsply DeTrey GmbH, Konstanz, Germany). As control, the orthodontic adhesive system Transbond XT primer (3M Unitek) was used. The adhesives were employed based on the manufacturer's recommendations and polymerized using a LED light-curing unit (Valo; Ultradent, South Jordan, Utah, United States) with a light output of $1,000 \mathrm{~mW} / \mathrm{cm}^{2}$ ( - Table 1). In double application groups, one coat adhesive was applied and light-cured, then the second layer was applied similarly. After adhesive application, a light-cured orthodontic adhesive composite resin (Transbond XT; 3M Unitek) was applied to the base of stainless steel premolar brackets (MBT 0.22 slot diamond, Miniseries 2000 Ormco, United States) with a bracket base area of $10.29 \mathrm{~mm}^{2}$, and then the bracket was bonded at the center of the clinical crown by pressing tightly onto the buccal enamel surface. The surplus composite resin was distracted from bracket margin using a scaler. The light-curing was performed for 40 seconds ( 10 seconds from each side) at a distance of 1 to $2 \mathrm{~mm}$ of light tip from the bracket margins using the LED light-curing unit. The teeth were kept in distilled water for 24 hours at $37^{\circ} \mathrm{C}$ before testing.

Each specimen was placed on mounting jig in a universal testing machine (Autograph AGS-X; Shimadzu, Kyoto, Japan) with bracket base parallel to the shear load. A shear force for debonding was applied to bracket base in occluso-gingival direction at a crosshead speed of $1 \mathrm{~mm} /$ minute. The maximum force required to debonding of the bracket was recorded as Newton and calculated in MPa by dividing the imposed force (in Newton) at the time of fracture by the bracket base area (in $\mathrm{mm}^{2}$ ). After debonding, the enamel surfaces were examined with a stereomicroscope at $\times 20$ magnification to check site of bond failure and remaining adhesive on tooth using adhesive remnant index (ARI) as described by Årtun and Bergland. ${ }^{22}$ This index uses four scores-(0) no adhesive residue in bonding area on tooth, (1) less than $50 \%$ of the adhesive remaining in bonding area on the tooth, (2) more than $50 \%$ of the adhesive remaining in the bonding area on the tooth, and (3) all the adhesive remaining on the tooth in the bonding area.

Data of shear bond strengths were statistically evaluated with the SPSS Program, version 20.0 (Statistical Package for the Social Sciences; SPSS, Chicago, Illinois, United States). The normal distribution of data was confirmed by Kolmogorov-Smirnov test. The data were analyzed using one-way analysis of variance (ANOVA), followed by post hoc least significant difference (LSD) tests to compare the means between groups. The data of ARI scores were submitted to chi-squared test. $p$-Value of less than 0.05 was considered statistically significant for all statistical analyses. 
Table 1 Adhesive systems used, chemical composition, and application procedure

\begin{tabular}{|c|c|c|}
\hline Adhesive systems & Composition & Application procedure \\
\hline $\begin{array}{l}\text { Scotchbond Universal } \\
\text { (3M Oral Care, St. Paul, } \\
\text { Minnesota, United States) } \\
\text { Lot no: } 602724\end{array}$ & $\begin{array}{l}\text { 10-MDP phosphate monomer, } \\
\text { dimethacrylate resins, HEMA, } \\
\text { methacrylate-modified polyalkenoic } \\
\text { acid copolymer, filler, ethanol, water, } \\
\text { initiators, silane }\end{array}$ & $\begin{array}{l}\text { 1. Apply the adhesive to the entire preparation with a micro- } \\
\text { brush and rub it in for } 20 \mathrm{~s} \\
\text { 2. Direct a gentle stream of air over the liquid for about } 5 \\
\text { s until it no longer moves and the solvent is evaporated } \\
\text { completely } \\
\text { 3. Light-cure for } 10 \mathrm{~s}\end{array}$ \\
\hline $\begin{array}{l}\text { Prime\&Bond Universal } \\
\text { (Dentsply DeTrey GmbH, } \\
\text { Konstanz, Germany) } \\
\text { Lot no: } 1802000551\end{array}$ & $\begin{array}{l}\text { HEMA, 2-hydroxy-3 acryloyloxypropyl } \\
\text { methacrylate, UDMA, trimethylol- } \\
\text { propane trimethacrylate, PENTA, } \\
\text { diketone, organic phosphine oxide, } \\
\text { stabilizers, cetylamine hydrofluoride, } \\
\text { acetone, water }\end{array}$ & $\begin{array}{l}\text { 1. Apply the adhesive to air-dried enamel/dentin surface with } \\
\text { rubbing for } 20 \mathrm{~s} \\
\text { 2. Gentle stream of air applied over the liquid for at least } 5 \mathrm{~s} \\
\text { 3. Light-cure for } 10 \mathrm{~s}\end{array}$ \\
\hline $\begin{array}{l}\text { Transbond XT } \\
\text { (3M Unitek, St. Paul, } \\
\text { Minnesota, United States) Lot } \\
\text { no: N884766 }\end{array}$ & $\begin{array}{l}\text { Bis-GMA, TEGDMA, } \\
\text { 4-(dimetylamino)-benzene ethanol, } \\
\text { DL-camphorquinone, hydroquinone }\end{array}$ & $\begin{array}{l}\text { 1. Apply Transbond XT etching gel to tooth surface for } 15 \mathrm{~s} \\
\text { 2. Rinse thoroughly with water to ensure total removal of } \\
\text { etchant } \\
\text { 3. Apply thin uniform coat of Transbond XT primer }\end{array}$ \\
\hline
\end{tabular}

Abbreviations: 10-MDP, 10-methacryloyloxydecyl dihydrogen phosphate; Bis-GMA, bisphenol-glycidyl methacrylate; HEMA, hydroxyethylmethacrylate; PENTA, dipentaerythritol penta acrylate monophosphate; TEGDMA, triethylene glycol dimethacrylate; UDMA, urethane dimethacrylate.

Note: Composition as provided by the manufacturers.

Table 2 One-way ANOVA results for shear bond strength test

\begin{tabular}{|l|l|l|l|l|l|}
\hline & Sum of squares & df & Mean square & $\boldsymbol{F}$ & $\boldsymbol{P}$ \\
\hline Between groups & 501.253 & 4 & 125.313 & 14.928 & $0.000^{\mathrm{a}}$ \\
\hline Within groups & 587.634 & 70 & 8.395 & & \\
\hline Total & 1088.887 & 74 & & & \\
\hline
\end{tabular}

Abbreviations: ANOVA, analysis of variance; $\mathrm{df}$, degrees of freedom.

astatistically significant differences $(p<0.05)$.

Table 3 Mean shear bond strengths of the different experimental groups

\begin{tabular}{|l|l|}
\hline Experimental groups & MPa \pm SD \\
\hline Scotchbond Universal & $11.38 \pm 3.25^{\mathrm{a}}$ \\
\hline Scotchbond Universal-Double application & $11.92 \pm 3.88^{\mathrm{a}}$ \\
\hline Prime\&Bond Universal & $4.92 \pm 1.24^{\mathrm{b}}$ \\
\hline Prime\&Bond Universal-Double application & $8.25 \pm 2.89^{\mathrm{c}}$ \\
\hline Transbond XT & $7.52 \pm 2.55^{\mathrm{c}}$ \\
\hline
\end{tabular}

Abbreviation: SD, standard deviation.

Note: Same superscript small letter indicates no statistically significant difference in the columns.

\section{Results}

The one-way ANOVA disclosed statistically significant differences between the experimental groups (-Table 2). The mean shear bond strengths and standard deviations are shown in - Table $\mathbf{3}$ and -Fig. $\mathbf{1}$ including the results of multiple comparisons by LSD post hoc test. The highest shear bond strengths were acquired by Scotchbond Universal $(p<0.05)$. The double application of Scotchbond Universal did not affect the shear bond strength $(p>0.005)$. The lowest shear bond strength was found in the Prime\&Bond Universal
Table 4 Distribution of ARI scores

\begin{tabular}{|l|l|l|l|l|l|}
\hline Experimental groups & $\mathbf{0}$ & $\mathbf{1}$ & $\mathbf{2}$ & $\mathbf{3}$ & $\mathbf{p}$ \\
\hline Scotchbond Universal & 5 & 4 & 2 & 4 & \\
\hline $\begin{array}{l}\text { Scotchbond Universal- } \\
\text { Double application }\end{array}$ & 5 & 3 & 3 & 4 & \\
\hline Prime\&Bond Universal & 9 & 4 & 2 & 0 & $0.868^{\text {a }}$ \\
\hline $\begin{array}{l}\text { Prime\&Bond Universal- } \\
\text { Double application }\end{array}$ & 6 & 4 & 3 & 2 & \\
\hline Transbond XT & 6 & 3 & 3 & 3 & \\
\hline
\end{tabular}

Abbreviation: ARI, adhesive remnant index.

aStatistically not significant differences $(p<0.05)$.

group $(p<0.05)$. The double application of Prime\&Bond Universal increased the shear bond strength $(p<0.05)$.

The distribution of ARI scores is shown in - Table 4 and -Fig. 2. The evaluation of the ARI scores by chi-squared test revealed no statistically significant difference in the distribution of scores between groups $(p>0.05)$.

\section{Discussion}

The clinicians have been very successful in the "direct bonding method" for nearly 40 years since the 1970s. With the 


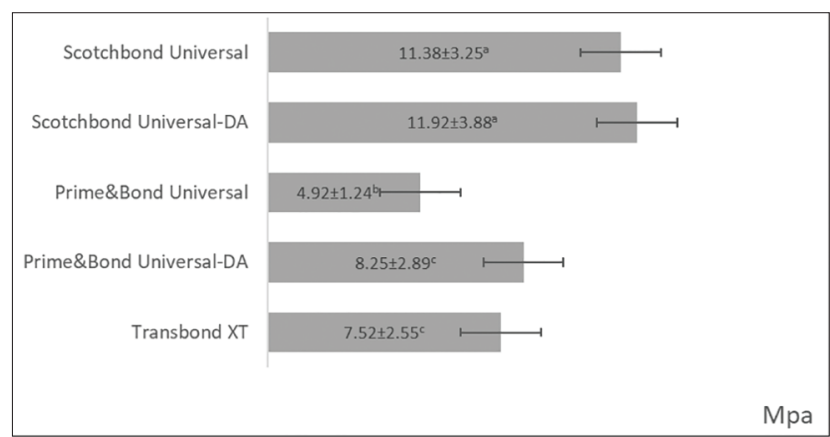

Fig. 1 Shear bond strengths (MPa \pm standard deviation [SD]) of the different experimental groups. Means and standard deviations are represented inside the bars. Means with the same superscript are not significantly different from each other (DA, double application).

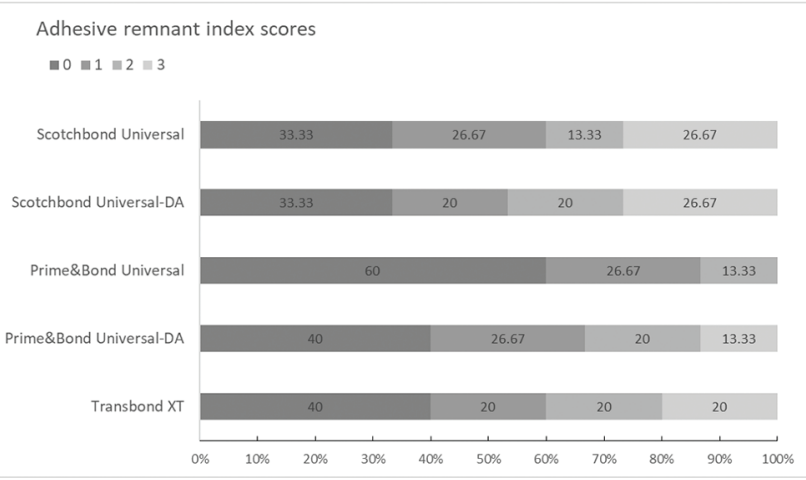

Fig. 2 The frequencies of adhesive remnant index (ARI) scores (\%) observed using light microscopy (DA, double application).

development of the direct bonding technique, the time spent in the clinic for bracket bonding has shortened, thus obtaining a more aesthetic and hygienic orthodontic treatment. ${ }^{23}$ Nowadays, the direct bonding technique with light-curing adhesives for bonding orthodontic brackets is widely used. The bonding strength of adhesives must be able to withstand the bite forces, the tension of the arch wires, and the patient's harmful mouth habits. According to Reynolds, ${ }^{24}$ the bonding strength is sufficient between 5.9 and $7.8 \mathrm{MPa}$ for orthodontic treatment. The shear bond strength of the bracket to enamel must be high enough for preventing bracket debonding during treatment, but it must not cause any enamel damage during debonding. ${ }^{2,5}$ It has also been reported that the enamel fractures might be formed when the shear bond strength was higher than $14 \mathrm{MPa} .^{25}$

Several generations of adhesive materials have been developed for restorative purposes and later been used for also orthodontic treatments. ${ }^{23}$ Dental adhesives are currently classified as "etch-and-rinse" and "self-etch" systems. ${ }^{7}$ In etch-and-rinse adhesives, the adhesion mechanism is mainly mechanical interlocking between demineralized dental hard tissue and a cured adhesive resin layer. Before the application of an etch-and-rinse adhesive, the phosphoric acid effectively demineralizes the dental hard tissues and contributes to adhesion via the penetration of resin monomers into dentinal tubules and the formation of a hybrid layer. ${ }^{7}$ The universal adhesives are the latest developed adhesives. ${ }^{8}$
Although the universal adhesives may be employed in both etch-and-rinse and self-etch modes, in this study, the universal adhesives were tested in etch-and-rinse mode. It has been previously reported that the application of an etching step prior to universal adhesives improved their bonding performance to enamel. ${ }^{8}$ It has also concluded that an acid etching for 15 seconds before universal adhesives increased bracket shear bond strength. ${ }^{6}$ In this study, the acid etching was performed for 15 seconds in all experimental groups. If the etching time prolongs, the frequency of enamel fracture might increase. ${ }^{5,6}$

In the present study, all shear bond strengths were lower than $14 \mathrm{MPa}$. Scotchbond Universal provided the higher shear bond strength than Transbond XT. The lower shear bond strength was obtained with Prime\&Bond Universal than Transbond XT. Therefore, the null hypothesis, that there would not be significant differences in shear bond strength between the universal adhesives and Transbond XT was rejected. The difference in bond strengths could be due to the functional monomers of the universal adhesives, as they are different. The performance of adhesives that have self-etching ability is related to their functional monomer content. ${ }^{26}$ The universal adhesives are single-component and one-step adhesives, which involve functional resin monomers that can promote chemical and micromechanical adhesion to the dental hard tissues. ${ }^{8}$ Scotchbond Universal includes 10-methacryloyloxydecyl dihydrogen phosphate (MDP) as the acidic functional monomer. 10-MDP is considered the most effective acidic functional monomer because it etches dentin, ionically bonds to calcium in hydroxyapatite, and forms stable nanolayered calcium salts. ${ }^{7,26}$ Scotchbond Universal also involves a polyalkenoic acid co-polymer, which can also bond chemically to hydroxyapatite. ${ }^{27}$ Nevertheless, it has been stated that the polyalkenoic acid co-polymer potentially contests with the 10-MDP functional monomer for calcium-bonding areas in hydroxyapatite, and may also inhibit monomer polymerization due to its high molecular weight. ${ }^{27}$ In agreement with this study, it has been concluded that Scotchbond Universal showed higher bond strength than Transbond XT, and this resulted from the 10-MDP monomer. ${ }^{6}$ The main functional monomer of Prime\&Bond Universal is PENTA. In a previous study, Prime\&Bond Universal presented similar bond strengths when compared with Scotchbond Universal. ${ }^{28}$ But, in this study, Scotchbond Universal provided higher shear bond strength than Prime\&Bond Universal. Furthermore, Scotchbond Universal includes filler particles which Prime\&Bond Universal did not have. The filler particles might contribute to high bonding performance, ${ }^{29}$ additionally high shear bond strength for orthodontic brackets. ${ }^{6,30}$

The thin adhesive layer thickness of universal adhesives might cause a decrease in the bond strength of universal adhesives. ${ }^{13}$ The enhanced bond strength of universal adhesives by the double application of universal adhesives and the application of an extra adhesive layer with these adhesives has been reported. ${ }^{13-16}$ This improved bond strength has been attributed to the formation of a thicker adhesive layer by the double application. The thicker adhesive layer 
has enhanced mechanical properties, thus reducing polymerization stresses and achieving stress-distribution during testing. ${ }^{15,18,21,29}$ However, it may be more difficult to volatilize the solvent from a thicker adhesive layer before light curing. ${ }^{14}$ The solvent in adhesive formulation must completely be evaporated because the residual solvent weakens the adhesive interface and reduces the bond strength. ${ }^{7}$ This problem could be overcome by light-curing the adhesive layer before the application of second layer. ${ }^{14}$ It has been reported that when the first layer is light-cured, the thickness of the adhesive layer could be increased by the application of second layer. Nevertheless, there is still no consensus about what the optimum thickness for the adhesive layer of each adhesive. Moreover, the double application might enhance the hardness of the adhesive layer, doing so improve the bond strength. ${ }^{15}$ The double application may also create a more uniform adhesive layer by compensating for probable application defects. ${ }^{16}$

In this study, the double application of Prime\&Bond Universal increased the shear bond strength, but the double application of Scotchbond Universal did not impact the shear bond strength. Therefore, the null hypothesis that the double application of universal adhesives would not improve the shear bond strength of orthodontic brackets was partially rejected. The improved bond strength of Prime\&Bond Universal could be due to the increasing of adhesive layer thickness by double application. Scotchbond Universal provided adequate bond strength by a single application, and the bond strength was not affected after double application. It could result from that Scotchbond Universal contains filler particles. It has been stated that the filler containing adhesives might form a sufficient thick adhesive layer by a single application, and the double application could not affect the bond strength of these adhesives. ${ }^{17,29}$

The high shear strength values of orthodontic brackets might be associated with high amounts of adhesive remaining on the enamel surface. ${ }^{1,6}$ However, in the present study, there was no statistically significant difference among the ARI values of the groups. The high or low ARI score may depend not only on the shear bond strength but also on many factors, such as the content of the adhesive, the base design of the brackets, and the properties of the prepared enamel, ${ }^{6}$ therefore the ARI values may not exactly represent bond strength. ${ }^{6}$ The tested adhesives revealed more an ARI value of 0 , which indicates that there is no residual adhesive or too little on the tooth surfaces so that the residual adhesive may easily be removed without sacrificing dental enamel. Furthermore, it can also be said that the tested adhesives are safe for clinical use because no enamel cracks or fractures were detected on the tooth surfaces.

\section{Conclusion}

Under the limitations of this in vitro study, the higher bond strength was obtained with Scotchbond Universal than Transbond XT. The double application of Prime\&Bond Universal provided a similar bond strength with Transbond XT. The universal adhesives might be an alternative for the bonding of orthodontic brackets. The use of universal adhesives in orthodontic treatment may be beneficial decreasing the number of required adhesive systems in dental clinics. Nonetheless, further laboratory studies must be conducted, and clinical studies are necessary to confirm the results.

\section{Conflict of Interest}

There are no conflicts of interest.

\section{References}

1 Türköz C, Ulusoy C. Evaluation of different enamel conditioning techniques for orthodontic bonding. Korean J Orthod 2012;42(1):32-38

2 Hellak A, Rusdea P, Schauseil M, Stein S, Korbmacher-Steiner HM. Enamel shear bond strength of two orthodontic self-etching bonding systems compared to Transbond $^{\mathrm{TM}}$ XT. J Orofac Orthop 2016;77(6):391-399

3 Cerone M, El-Badrawy W, Gong SG, Prakki A. Bond strength of universal self-etch 1-step adhesive systems for orthodontic brackets. J Can Dent Assoc 2019;85:j6

4 Malik SA, Laxmikanth SM. Comparative evaluation of the shear bond strength and de-bonding properties of self-etch adhesive composite cement (Maxcem Elite Chroma) vs. self-etching primer composite system (Transbond Plus Sep, 3M Unitek, Monrovia, Calif) used for orthodontic bracket bonding. Dentist 2019;1:1007

5 Scougall Vilchis RJ, Yamamoto S, Kitai N, Yamamoto K. Shear bond strength of orthodontic brackets bonded with different self-etching adhesives. Am J Orthod Dentofacial Orthop 2009;136(3):425-430

6 Shafiei F, Sardarian A, Fekrazad R, Farjood A. Comparison of shear bond strength of orthodontic brackets bonded with a universal adhesive using different etching methods. Dental Press J Orthod 2019;24(4):33.e1-33.e8

7 Van Meerbeek B, Yoshihara K, Van Landuyt K, Yoshida Y, Peumans M. From Buonocore's pioneering acid-etch technique to self-adhering restoratives. A status perspective of rapidly advancing dental adhesive technology. J Adhes Dent 2020;22(1):7-34

8 Cuevas-Suárez CE, da Rosa WLO, Lund RG, da Silva AF, Piva E. Bonding performance of universal adhesives: an updated systematic review and meta-analysis. J Adhes Dent 2019;21(1):7-26

9 Tahmasbi S, Badiee M, Modarresi M. Shear bond strength of orthodontic brackets to composite restorations using universal adhesive. J Dent (Shiraz) 2019;20(2):75-82

10 Naseh R, Afshari M, Shafiei F, Rahnamoon N. Shear bond strength of metal brackets to ceramic surfaces using a universal bonding resin. J Clin Exp Dent 2018;10(8):e739-e745

11 Lee J-Y, Ahn J, An SI, Park J-W. Comparison of bond strengths of ceramic brackets bonded to zirconia surfaces using different zirconia primers and a universal adhesive. Restor Dent Endod 2018;43(1):e7

12 Golshah A, Mohamadi N, Rahimi F, Pouyanfar H, Tabaii ES, Imani MM. Shear bond strength of metal brackets to porcelain using a universal adhesive. Med Arh 2018;72(6):425-429

13 Ahmed MH, De Munck J, Van Landuyt K, Peumans M, Yoshihara K, Van Meerbeek B. Do universal adhesives benefit from an extra bonding layer? J Adhes Dent 2019;21(2):117-132

14 Ermis RB, Ugurlu M, Ahmed MH. Van Meerbeek B. Universal adhesives benefit from an extra hydrophobic adhesive layer when lightcured beforehand.JAdhes Dent 2019;21(2):179-188

15 Chowdhury AFM, Saikaew P, Alam A, Sun J, Carvalho RM, Sano H. Effects of double application of contemporary self-etch 
adhesives on their bonding performance to dentin with clinically relevant smear layers. J Adhes Dent 2019;21(1):59-66

16 Fujiwara S, Takamizawa T, Barkmeier WW, et al. Effect of double-layer application on bond quality of adhesive systems. J Mech Behav Biomed Mater 2018;77:501-509

17 de Silva AL, Lima DA, de Souza GM, dos Santos CT, Paulillo LA. Influence of additional adhesive application on the microtensile bond strength of adhesive systems. Oper Dent 2006;31(5):562-568

18 Reis A, Albuquerque M, Pegoraro M, et al. Can the durability of one-step self-etch adhesives be improved by double application or by an extra layer of hydrophobic resin? J Dent 2008;36(5):309-315

19 Ito S, Tay FR, Hashimoto M, et al. Effects of multiple coatings of two all-in-one adhesives on dentin bonding. J Adhes Dent 2005;7(2):133-141

20 Albuquerque M, Pegoraro M, Mattei G, Reis A, Loguercio AD. Effect of double-application or the application of a hydrophobic layer for improved efficacy of one-step self-etch systems in enamel and dentin. Oper Dent 2008;33(5):564-570

21 Choi KK, Condon JR, Ferracane JL. The effects of adhesive thickness on polymerization contraction stress of composite. J Dent Res 2000;79(3):812-817

22 Årtun J, Bergland S. Clinical trials with crystal growth conditioning as an alternative to acid-etch enamel pretreatment. Am J Orthod 1984;85(4):333-340
23 Harari D, Aunni E, Gillis I, Redlich M. A new multipurpose dental adhesive for orthodontic use: an in vitro bond-strength study. Am J Orthod Dentofacial Orthop 2000;118(3):307-310

24 Reynolds IR. A review of direct orthodontic bonding. $\mathrm{Br}$ J Orthod 1975;2:171-178

25 Eminkahyagil N, Arman A, Cetinşahin A, Karabulut E. Effect of resin-removal methods on enamel and shear bond strength of rebonded brackets. Angle Orthod 2006;76(2):314-321

26 Yoshida Y, Nagakane K, Fukuda R, et al. Comparative study on adhesive performance of functional monomers. J Dent Res 2004;83(6):454-458

27 Wagner A, Wendler M, Petschelt A, Belli R, Lohbauer U. Bonding performance of universal adhesives in different etching modes. J Dent 2014;42(7):800-807

28 Bacelar-Sá R, Giannini M, Ambrosano GMB, Bedran-Russo AK. Dentin sealing and bond strength evaluation of hema-free and multi-mode adhesives to biomodified dentin. Braz Dent J 2017;28(6):731-737

29 Taschner $M$, Kümmerling $M$, Lohbauer $U$, Breschi L, Petschelt A, Frankenberger R. Effect of double-layer application on dentin bond durability of one-step self-etch adhesives. Oper Dent 2014;39(4):416-426

30 Boruziniat A, Khazaei Y, Motaghi S, Moghaddas M. Evaluation of bond strength of orthodontic brackets without enamel etching. J Clin Exp Dent 2015;7(4):e519-e523 